\title{
Résumés des POSTERS primés et présentés en communications orales au XXVème Congrès de la SALF
}

\author{
10-11 octobre 2008, Hammamet, Tunisie
}

\section{POSTER 1}

Intérêt de l'étude de l'oxydation de l'ADN des spermatozoïdes par marquage de la 8-oxoguanine en cytométrie en flux chez l'homme infertile

\author{
N. CHAKROUN FEKI 1 , N. ZRIBI ${ }^{1}$, H. ELEUCH ${ }^{2}$, R. GDOURA ${ }^{3}$, \\ A. SELLAMI ${ }^{1}$, A. BAHLOUL ${ }^{4}$, A. HAMMAMI ${ }^{3}$, J. GARGOURI ${ }^{2}$, \\ T. REBAI ${ }^{1}$, L. KESKES AMMAR ${ }^{1}$ \\ 1 Laboratoire d'Histologie Embryologie, \\ Faculté de Médecine de Sfax, Tunisie \\ 2 Centre de transfusion sanguine, Sfax \\ 3 Laboratoire de Microbiologie, \\ Faculté de Médecine de Sfax, Tunisie \\ 4 Unité de recherche infertilité masculine UR07US0011 \\ nozhafeki@yahoo.fr lkeskes@yahoo.fr
}

Introduction : Dans le sperme, le stress oxydatif est du à une production excessive de dérivés actifs de l'oxygène (DAO) d'origine essentiellement leucocytaire. L'oxydation de l'ADN est due à l'action directe des DAO sur l'ADN qui induit la formation de plusieurs adduits dont le plus étudiée est la 8-oxoguanine. L'intégrité de l'ADN est essentielle pour la fécondance du spermatozoïde et elle constitue de nos jours un sujet d'intérêt pour les chercheurs et cliniciens dans le monde. Si les moyens d'évaluation de l'intégrité globale de l'ADN spermatique se sont bien développés ces demières années, les tests d'évaluation du dommage de l'ADN d'origine oxydative sont peu documentés. L'objectif de ce travail est d'évaluer l'oxydation de l'ADN par marquage du 8-oxo-guanine en cytométrie en flux chez l'homme infertile.

Matériel et méthodes : Notre travail a porté sur 15 échantillons de sperme qui ont fait l'objet d'une analyse spermiologique selon les recommandations de l'OMS, avec une mesure de la concentration des leucocytes par la méthode cytochimique révélant la peroxydase dans les granulations cytoplasmiques. L'étude de l'oxydation de l'ADN a été faite par un kit de marquage de la 8-oxo-guanine en cytométrie de flux.

Résultats : Nous avons montré par l'étude de la régression linéaire une forte corrélation entre l'oxydation de l'ADN et le taux de leucocytes dans le sperme $(p=0,006, r=0,7)$. Un seuil de 250000 leucocytes $/ \mathrm{ml}$ de sperme était associé à une augmentation significative de l'oxydation de l'ADN $(p=0,03)$.
Conclusion : Nos résultats montrent que la 8-oxoguanine pourrait être considérée comme un biomarqueur de l'action directe du stress oxydatif sur l'ADN. Dans le sperme, l'ADN spermatique semble être vulnérable à des taux relativement faibles de DAO produits par des leucocytes présents à une concentration largement inférieure au seuil de la leucospermie définie par l'OMS ( $\geq 1 \mathrm{million} / \mathrm{ml}$ ).

\section{POSTER 2}

Anomalies chromosomiques constitutionnelles et spermatiques chez les patients Tunisiens atteints de troubles non obstructifs de la spermatogenèse

\section{H. ELGHEZAL, M. MEHDI, M. MAHJOUB, S. MOUGOU, S. IBALA, M. AJINA, A. SAAD}

Service de Cytogénétique et de Biologie de la Reproduction. CHU Farhat Hached - Sousse - Tunisie hatem_elghezal@yahoo.fr

Introduction : Les analyses cytogénétiques présentent un intérêt particulier dans l'exploration des altérations de la spermatogenèse. En effet, les anomalies chromosomiques constituent une cause majeure des troubles de la production des spermatozoïdes. Dans un autre sens, un accident de la méiose peut provoquer chez un homme à caryotype constitutionnel normal, la production de spermatozoïdes de constitution chromosomique déséquilibrée. Nous rapportons dans ce travail les résultats des différentes explorations cytogénétiques réalisées sur une large série de patients Tunisiens atteints de troubles non obstructifs de la spermatogenèse.

Matériel et Méthodes : 1100 patients atteints de différents troubles non obstructifs de la spermatogenèse sont inclus dans cette étude. Une étude du caryotype constitutionnel sur culture de lymphocytes sanguins est réalisée chez l'ensemble de ces patients. Des explorations par Hybridation In Situ Fluorescente (FISH) sont réalisées sur des patients atteints d'azoospermie avec un caryotype normal ainsi que des patients atteints de syndrome de Klinefelter avec une spermatogenèse résiduelle à la recherche de faible mosaïcisme 46,XY/47,XXY.

La technique de FISH sur spermatozoïdes est réalisée sur des échantillons de patients atteints d'oligozoospermie sévère, de tératozoospermie ainsi que des patients qui présentent un remaniement chromosomique constitutionnel, pour évaluer le taux 
de spermatozoîdes déséquilibrés par rapport aux témoins.

Résultats : L'étude des caryotypes montre la présence de 142 anomalies chromosomiques $(12.91 \%)$ réparties en 84 anomalies parmi 371 cas d'azoospermie (22.64\%), 27 anomalies parmi 334 cas d'oligozoospermie extrême (8.08\%), 19 anomalies parmi 208 cas d'oligozoospermie sévère $(9.13 \%)$ et 12 anomalies chez les 144 patients atteints d'oligozoospermie modérée (8.33\%). Aucune anomalie n'est détectée dans le groupe des 43 tératozoospermies. La FISH sur lymphocytes retrouve parmi 35 patients azoospermiques à caryotype normal, 4 cas de mosaïcisme $X Y / X X Y$ et un cas de mosaïcisme $X Y / X X Y / X X X Y$. La même technique utilisée chez 12 patients atteints du syndrome de Klinefelter montre la présence de mosaïcisme chez les patients oligospermiques et l'absence de ce mosaïcisme chez les patients azoospermiques.

La FISH sur spermatozoïdes montre un taux élevé de mal ségrégations chromosomiques chez les patients atteints d'oligozoospermie sévère et de tératozoospermie par rapport aux témoins. Les spermatozoïdes des patients atteints de translocation robertsonienne $(13 ; 14)$ montre un taux de déséquilibrés qui varie entre $18.30 \%$ à $37.84 \%$ sans aucune corrélation avec les paramètres spermatiques.

Discussion et conclusion : Nos résultats confirment la forte prévalence des anomalies chromosomiques chez les hommes atteints de troubles de la spermatogenèse. La présence des anomalies essentiellement autosomiques dans le groupe d'homme à phénotype atténué doit être prise en considération du fait du risque élevé de produire des zygotes déséquilibrés. D'autre part, et en dehors de toute anomalie chromosomique constitutionnelle, les altérations méiotiques associées à l'oligozoospermie et à la tératozoospermie paraissent favoriser les accidents de non disjonction chromosomique et augmenter, par conséquent, la fréquence de production de spermatozoïdes déséquilibrés.

\section{POSTER 3}

Stratégie de la prise en charge de l'azoospermie à la clinique BIASA de Lomé (TOGO)

\author{
M. FIADJOE* , A.K. ADUAYI*, K. KOLANI*, B. KOLANI*, \\ M. BAILLY**, R. BOULANGER ${ }^{\star \star *}$ \\ * Centre de fertilité. Clinique BIASA Lomé TOGO \\ ** Centre d'AMP du CHI Roissy. FRANCE \\ *** Centre d'AMP de la clinique de Cormeilles en Parisis. France \\ mfiadjoe@hotmail.com
}

Introduction : En Afrique, l'annonce de l'azoospermie est un véritable drame social. Les nouvelles techniques d'AMP ont suscité de grands espoirs pour permettre à ces hommes d'avoir des enfants qui leur sont génétiquement liés. Les objectifs de cette étude sont de deux ordres :

- d'abord, tester la technique d'exploration épididymo-testiculaire (EET) dans le contexte d'un pays médicalement peu développé

- puis, envisager dans la mesure du possible, un schéma simplifié de prise en charge de l'azoospermie.

Matériel et Méthodes : II s' agit d'une étude rétrospective qui a recensé tous les cas d' azoospermie pris en charge à la clinique BIASA de Lomé depuis le début de ses activités de 01-05-1984 jusqu'au 30-04-2008. Les différents moyens d'exploration ainsi que les traitements proposés ont été répertoriés et les résultats notés.
Résultats : Au total 114 cas ont été colligés sur deux périodes :

* De 1984 à 1997 : 38 cas. Nous n'avions que l'IAD comme solution; la biopsie testiculaire était à visée pronostique. 19 biopsies testiculaires se sont avérées négatives. 10 hommes sur 19 (53\%) ont accepté une IAD dont 7 ont abouti à une naissance vivante.

* De 1998 à 2008 : 76 cas. La prise en charge passe par une EET en vue d'une ICSI réalisée en asynchrone. Les critères d'élection à cette EET sont essentiellement les marqueurs cliniques (taille des testicules selon l'orchidomètre de PRADER) et hormonaux (dosage de la FSH). L'EET a été réalisée chez 28 azoospermes (37\%) et a été positive dans $67 \%$ (19cas). L'ICSI a été réalisée pour $12(43 \%)$ couples dont $5(42 \%)$ ont eu une grossesse. Deux enfants $(17 \%)$ en sont nés, $1(8 \%)$ GEU et $2(17 \%)$ fausses couches ont été enregistrées.

Discussion et conclusion : Le grand défi était de définir des critères simples permettant de faire la différence entre une azoospermie sécrétoire (non obstructive) et une azoospermie excrétoire (obstructive). Ne disposant pas de moyens d'exploration poussée (marqueurs séminaux, déférentographie, cytogénétiques etc.), nous nous basons sur le volume testiculaire et le taux de FSH et avons ainsi défini une valeur prédictive de recueil positif de spermatozoïdes lors de l'EET : FSH $\times 10$ - volume testiculaire $\leq$ 120. Lorsque la valeur prédictive est défavorable, d'autres solutions sont proposées : IAD, adoption... Malgré le coup élevé non seulement de l'installation mais surtout de la prestation, l'acceptabilité est grande eu égard à la résolution du problème éthique et d'honneur de la famille. L'EET vient redonner de nouveaux espoirs à condition qu'elle tienne compte de certains critères d'éligibilité dont la règle de 120 qui reste à être validée par d'autres études.

\section{POSTER 4}

Infertilité masculine chez les patients normospermiques : analyse protéomique des spermes normaux non fécondants en fécondation in vitro classique

C. FRAPSAUCE1, C. PIONNEAU2, J. Bouley3, V. DE LAROUZIĖRE1, I. BERTHAUT1, C. RAVEL1, J.M. ANTOINE4, F. SOUBRIER3, J. MANDELBAUM1

1 Service d'Histologie et de Biologie de la Reproduction Université Pierre et Marie Curie (Paris VI) - EA 1533- Hôpital Tenon (APHP), Paris 20 e.

2 Plateforme post-génomique de la Pitié-Salpêtrière (P3S) Université Pierre et Marie Curie (Paris VI)

3 Unité Inserm U525 - Pitié-Salpêtrière, Paris 13e

4 Service de Gynécologie, Obstétrique et Médecine de la Reproduction - Hôpital Tenon, Paris 20e c_frapsauce@yahoo.fr

Au cours des fécondations in vitro (FIV) classiques, $5 \%$ des tentatives se soldent par un échec de fécondation imprévisible, du fait d'une absence d'anomalies des paramètres spermatiques. Dans $56 \%$ des cas, aucune cause ovocytaire évidente n'est retrouvée et l'on observe, par contre, un défaut ou une absence de fixation des spermatozoïdes à la zone pellucide. Ce phénotype est similaire à celui décrit chez les souris invalidées pour le gène de l'enzyme de conversion de l'angiotensine I (ACE) germinale [1,2]. Sur la base des travaux chez l'animal et des constatations en FIV humaine, nous testons l'hypothèse physiopathologique selon laquelle les échecs de fécondation à sperme normal pourraient être liés à des déficits qualitatifs et/ou quantitatifs de la fonction enzymatique du spermatozoïde. 
Les sujets cas sont inclus devant un échec de fécondation en FIV classique et la visualisation d'un défaut de fixation des spermatozoïdes à la zone pellucide de l'ovocyte. Les sujets témoins sont des hommes à sperme normal et fécondant, participant à une FIV d'indication tubaire. Une étude cas-témoin de la fonction enzymatique de l'ACE germinale a déjà été entreprise lors d'un travail préliminaire et n'a pas montré de différence significative, concernant cette enzyme. L'étude a par conséquent été élargie en appliquant, sur les échantillons spermatiques, des techniques de protéomique, afin de comparer les profils d'expression protéique des spermatozoïdes entre les cas et les témoins à la recherche d'une éventuelle protéine différentiellement exprimée au niveau du gamète mâle.

Les échantillons de sperme ont été analysés par la technologie innovante d'électrophorèse 2D-DIGE avec au préalable une étape de pré-fractionnement des extraits protéiques en fonction de leur point isoélectrique $(\mathrm{pl})$. Cette méthode a permis la détection de 1486 spots protéiques sur la fraction acide ( $\mathrm{pH} 4.5-5.5)$ et de 2360 spots protéiques sur la fraction basique $(\mathrm{pH}$ 6-9). Huit protéines différentiellement présentes entre les cas et les témoins ont été mises en évidence dans la fraction acide et sept dans la fraction basique. Parmi ces protéines candidates, trois ont pu être identifiées par spectrométrie de masse. II s'agit de la glyoxalase 1 et de l'inhibiteur de la ribonucléase (protéines aux propriétés antioxydantes), et de la p40 ribosomal protein. Cette dernière protéine correspond à la même entité que le récepteur à la laminine (LR67). LR67 est connu pour interagir avec les intégrines, en facilitant la fixation de la laminine aux intégrines. LR67, étant augmenté chez les patients infertiles de cette étude, cela pourrait entraîner un défaut de fixation aux intégrines de certaines protéines impliquées dans l'interaction gamétique, au profit d'une fixation à la laminine. Cette hypothèse constitue une piste pouvant expliquer l'infertilité chez ces patients.

Des investigations seront menées pour déterminer si des anomalies d'expression des protéines identifiées en protéomique pourraient expliquer les échecs de fécondation de ces sujets, contribuant ainsi à préciser les causes moléculaires de certaines infertilités masculines non expliquées par les données de la spermiologie classique.

Références bibliographiques :

1 Krege J.H et al. Nature, 1995.375:146-8

2 Esther C.R. et al. Lab Invest, 1996.74:953-6

\section{POSTER 5}

Mise au point d'un modèle d'étude de la mélose in vitro chez le rat. Application en toxicologie : impact du chrome sur la spermatogenèse

\section{GEOFFROY-SIRAUDIN ${ }^{\text {ad }}$, R. GHALAMOUN-SLAIMIba, A. BOTTAA ${ }^{A}$, P. DURANDC, M. GUICHAOUA ${ }^{\text {ad }}$}

a Laboratoire de Biogénotoxicologie et Mutagenèse

Environnementale (EA1784), Aix-Marseille Université, Fédération de Recherche 3098, ECCOREV, Marseille, France

b Service d'histologie-Embryologie, Faculté de Médecine d'Oran, Université d'Oran, Algérie

c Unité INSERM/INRA U418, Institut de Génomique Fonctionnelle de Lyon, UMR 5242, Ecole Normale Supérieure de Lyon, France

d Laboratoire de Biologie de la reproduction. Hôpital de la Conception, Marseille, France cendrine.geoffroy@ap-hm.fr

Introduction : Depuis les travaux de Carisen en 1992, de nombreuses études ont montré une diminution significative de la qualité du sperme chez les hommes fertiles ou infertiles. L'exposition à des toxiques environnementaux est aujourd'hui considérée comme au moins en partie responsable de cette atteinte de la fonction de reproduction. La réglementation $\mathrm{REACH}$ impose des études reprotoxiques pour les produits mis sur le marché à plus de 100 tonnes par an. Afin d'évaluer l'impact de toxiques environnementaux et/ou professionnels sur la reproduction humaine tout en maîtrisant le nombre d'animaux nécessaires aux études, il est aujourd'hui nécessaire de développer de nouveaux modèles d'études in vitro. Le but de ce travail a été de mettre au point un modèle innovant permettant des études toxicologiques dans l'infertilité masculine puis d'étudier l'effet d'un métal lourd, le chrome, sur le déroulement de la méiose.

Matériel et méthodes : Nous avons utilisé des cultures de tubes séminifères de rat. Deux cultures ont tout d'abord été réalisées en présence de BrdU afin de valider la cinétique de différenciation des cellules dans ce modèle. Puis 3 séries de cultures ont été réalisées sans BrdU et à différentes doses de chrome : témoin, 1, 10 et 100 $\mathrm{mg} / \mathrm{L}$. L'impact de ce toxique sur les cellules germinales a été étudiè par immunocytochimie avec l'anticorps anti-SCP3. Nous avons analysé la richesse des étalements, la chronologie d'apparition des différents stades de la prophase I de méiose, ainsi que les trois anomalies les plus fréquemment retrouvées au cours de la méiose: asynapis, fragmentation des complexes synaptonémaux (CS), images en pointillés.

Résultats : L'analyse en immunocytochimie avec un double marquage SCP3-BRDU permet d'établir le profil de différenciation des cellules germinales en culture. Les concentrations élevées de chrome (10 et $100 \mathrm{mg} / \mathrm{L}$ ) provoquent une diminution de la richesse en cellules germinales et une desquamation des cellules de Sertoli s'accentuant au cours de la culture. La chronologie de différenciation des cellules méiotiques du rat ne semble pas affectée par l'ajout de chrome quelle que soit sa concentration. En présence de ce toxique, nous observons de façon dose-dépendante une augmentation très importante des taux d'asynapsis, et à moindre échelle du pourcentage de noyaux présentant une fragmentation des CS.

Discussion et Conclusion : Le chrome altère sévèrement le déroulement de la spermatogenèse en agissant à la fois sur les cellules germinales et sur les cellules de Sertoli. II pourrait agir directement sur la recombinaison et/ou l'appariement des chromosomes, mais aussi sur le micro-environnement testiculaire responsable secondairement de lésions des CS.

\section{POSTER 6}

Blessures dans la filiation et infertilité masculine

\author{
M. JAOUL1,2, M. ALBERT2,3, M. BAILLY2, D MOLINA \\ GOMES2, R. WAINER2, M. BERGERE2,3, J. SELVA2,3 \\ 1 Centre Clinique de Psychothérapie ; 2 Unité d'AMP, CHIPS, \\ 78300 POISSY ; 3 EA2493, Université Versailles \\ Saint Quentin en Yvelines, 78 \\ malbert@chi-poissy-st-germain.fr;mojaoul@noos.fr
}

Introduction : Bien souvent la clinique a pu retenir notre attention sur la présence répétitive de singularités dans I'histoire familiale d'hommes souffrant d'infertilité, en particulier des décès précoces du père ou des ruptures de liens entre pères et grands-parents paternels, des mésententes avec le père, des tentatives de spoliation d'héritage. A partir de cette clinique, la question se pose du rôle 
joué par certains événements de vie, par des modes particuliers de filiation, ainsi que par des spécificités du fonctionnement familial : seraient-ils susceptibles, par la voie des identifications, de venir inhiber chez certains hommes la capacité à engendrer, à prendre place dans la filiation? Ces constatations sont-elles liées au mode de recrutement ou existe t-il un lien entre la présence de ces particularités et la survenue d'une infertilité ?

Matériel et Méthodes : Pour répondre à ces questions, nous avons comparé à l'aide d'une grille de filiation, validée sur trois générations, un groupe de 30 hommes souffrant d'infertilité primaire sécrétoire inclus dans un protocole d'ICSI et un groupe de 30 hommes fertiles vus à la maternité lors de la naissance de leur enfant.

Résultats et Discussion : Sur les 12 items de la grille, certains étaient significativement retrouvés plus fréquemment dans le groupe d'hommes infertiles :

- Item 6 : décès précoce du père ou grand père seuls $(p<0.05)$;

- item 6' : décès précoce des parents et grands-parents $(p<0.01)$

- item 7 : enfants élevés hors famille $(p<0.05)$;

- item 9' : rupture du lien père/fils en relation avec la séparation du couple parental $(p<0.01)$.

S'agissant du mode de relation père/fils, nous avons dégagé un item supplémentaire : rupture de lien/mésentente grave entre père et fils à la génération du père ou à celle du fils (hors décès), qui concernait 15 des 30 patients du groupe infertile (vs 7 des 30 hommes du groupe témoin $-p<0.05$ )

Enfin, dans notre population d'hommes infertiles, nous avons pu noter que les évènements intervenaient principalement dans la branche paternelle.

L'approche comparative a été complétée, pour la moitié seulement des hommes du groupe infertile (pour des raisons de faisabilité), par une approche psychodynamique comprenant un récit de vie, un questionnaire semi-directif portant sur les représentations liées à la paternité et aux relations avec les parents, et un test projectif. Ainsi, dans ce sous-groupe on peut noter que : (1) 11 sujets ont vécu une perturbation impliquant leur génération ou la génération des parents dans la lignée paternelle, (2) lorsqu'il s'agit de séparation précoce (deuil, séparation durable, abandon), elle implique le plus souvent les deux parents, la mort de la mère ou une séparation durable d'avec elle entraînant la séparation durable avec le père, (3) pour 4 sujets l'alcoolisme d'un parent, le plus souvent le père, est suivi d'un décès précoce ou d'une séparation (4) 3 sujets ont vécu les ruptures de lien (abandon, séparation, deuil) dans la lignée maternelle, à la génération de leur mère, (5) pour certains sujets, il y a un cumul des traumatismes, dans les deux lignées ou sur les deux générations.

La prise en charge en ICSI de ces patients a montré des taux de grossesse et d'accouchement beaucoup plus élevés. II s'agit certes d'effectifs réduits, mais ces résultats nous ont permis de faire l'hypothèse que la mise en récit de l'histoire familiale, dans le contexte d'un " transfert de recherche " a pu permettre une relance générationnelle.

En conclusion : pour certains hommes, les conflits et les deuils non résolus, réactivés par le projet de paternité, trouveraient à se manifester dans l'expression somato-psychique d'infertilité, à défaut d'avoir pu trouver leur expression dans le psychisme. Le corps servirait alors de lieu d'une mémoire sans mémoire, en attente d'un sens à interpréter.

\section{POSTER 7}

Tabac et fertilité : quel impact sur le spermogramme

\section{A. LOUATI, A. AYACHI, G. MERDESSI, A. ZHIOUA, M. FERCHIOU, F. ZHIOUA, S. MERIAH}

Service de Gynécologie Obstétrique de l'hôpital Aziza Othmana. TUNIS anis.louati@laposte.net

Introduction : L'exploration de la stérilité du couple comporte plusieurs étapes et examens successifs aussi bien chez l'homme que chez la femme. En effet, l'évaluation de la fertilité masculine comporte comme première investigation l'étude du sperme. Notre étude consiste à évaluer l'impact du tabac sur les caractéristiques spermatiques avec les principales anomalies rencontrées dans notre série de patients.

Matériel et Méthodes : C'est une étude rétrospective réalisée au service de gynéco-obstétrique de l'hôpital AZIZA OTHMANA sur une période de deux années (01 janvier 2006 - 31 décembre 2007) qui a permis de colliger 163 études complètes de sperme. Les spermogrammes étaient réalisés chez des couples consultant pour la première fois pour une prise en charge de stérilité.

Deux groupes de patients ont été étudiés : groupe $A(n=110)$ : patients tabagique et groupe $B(n=53)$ : patients non tabagique.

Aucun des patients n'a reçu de traitement antibiotique ou vitaminique avant la réalisation du spermogramme dans les 2 groupes.

Résultats : Les deux groupes étaient homogènes pour l'âge, la durée de stérilité ainsi que le délai d'abstinence.

Dans le groupe A, $44,8 \%$ des patients avaient un tabagisme supérieur à 10 pq/année).

Dans le groupe B, $50,94 \%$ des spermogrammes étaient normaux alors qu'il est uniquement de $36,36 \%$ dans le groupe $A$.

La concentration moyenne en spermatozoïdes était $52,39 \mathrm{M} / \mathrm{ml}$ dans le groupe B alors qu'elle est de $24,9 \mathrm{M} / \mathrm{ml}$ dans le groupe $A$.

La mobilité moyenne dans le groupe A était de $22,2 \%$ alors qu'elle est de $39,4 \%$ dans le groupe $B$.

La moyenne des formes typiques dans le groupe A était de 18,72\% alors qu'elle est de $54,66 \%$ dans le groupe $B$.

Conclusion : L'infertilité masculine devient de plus en plus responsable de la stérilité du couple. Les résultats de notre étude viennent renforcer les donnés de la littérature. En effet, l'arrêt du tabac doit être le premier traitement proposé dans le cadre de prise en charge de la fertilité. 


\section{POSTER 8}

Effet des phtalates sur le développement du testicule fœtal humain

V. MUCZYNSKI1, R. LAMBROT1, C. LECUREUIL1, D. MOISON1, H. COFFIGNY1, C. PAIRAULT1, G. ANGENARD1, R. FRYDMAN2, R. HABERT1, V. ROUILLER-FABRE 1

\section{INSERM U566 ; Université P7 ; DSV/iRCM/LDRG: CEA 18 route du panorama 92260 Fontenay aux Roses \\ 2. Service de Gynécologie Obstétrique ; \\ Hôpital Antoine Béclère ; Clamart vincent.muczynski@cea.fr}

Au cours des dernières décennies plusieurs études ont démontré chez I'homme une baisse de la production et de la qualité spermatique au cours du temps, une augmentation des cas de cancer testiculaire et des anomalies de développement des organes génitaux (revue dans Bay et al. 2006 ; Sharpe et al, 2006). Ces différentes anomalies pourraient être les symptômes d'une même pathologie, le syndrome de dysgénésie testiculaire (TDS). Ce syndrome serait le résultat d'altérations du développement testiculaire pendant la vie fœtale, survenues suite à l'exposition du fotus aux perturbateurs endocriniens parmi lesquels figurent les phtalates. Ces molécules, qui interviennent dans la fabrication d'un grand nombre de composés (peintures, cosmétiques, emballages plastiques), sont reconnus comme étant reprotoxiques chez le rat, notamment en diminuant la production de testostérone (Mylchreest et al. 2002 ; Fisher et al. 2003), et ceci dans des modèles d'expérimentation in vivo et in vitro. Chez l'homme, des études cliniques ont montré une corrélation entre les niveaux de phtalates dans les urines ou le lait maternel et différentes modifications d'indicateurs de la masculinisation (Swan et al. 2005 ; Main et al. 2006).

- Dans cette étude nous avons recherché les effets du MEHP (Mono-EthylHexyl-Phtalates) sur les différentes fonctions du testicule fœetal humain, et ce grâce à un modèle de culture organotypique développé dans notre laboratoire (Lambrot et al. 2006). La culture est réalisée durant 4 jours en présence ou non de MEHP pour des doses allant de $10^{-4}$ à $10^{-6} \mathrm{M}$.

- Les fotus issus d'Interruption Volontaire de Grossesse proviennent du Service de Gynécologie Obstétrique du Professeur René Frydman de l'hôpital $A$. Béclère à Clamart et sont âgés de 7 à 12 semaines de gestation, période critique pour le développement du testicule humain puisque c'est à ce moment que se mettent en place d'une part la production de testostérone indispensable à la masculinisation, et d'autre part le stock de cellules germinales.

- Nous avons montré que le MEHP n'affecte pas la fonction des cellules de Leydig quelque soit la dose de MEHP utilisée. En revanche, bien qu'il ne semble pas affecter leur nombre, le MEHP altère une des fonctions des cellules de Sertoli en diminuant le niveau d'ARNm de l'AMH. Le MEHP affecte également la lignée germinale avec une augmentation de l'apoptose des cellules germinales qui se traduit par une diminution importante de leur nombre.

- Cette étude est la première analyse expérimentale de l'effet du MEHP sur le testicule fœtal humain montrant un effet négatif sur les cellules germinales et cellules de Sertoli. En revanche, contrairement au modèle rat, le MEHP n'affecte pas les cellules de Leydig.

Références bibliographiques :

Bay, K., et al. (2006) : Best Pract Res Clin Endocrinol Metab 20(1) :7790.

Sharpe, R.M., et al. (2006) : Best Pract Res Clin Endocrinol Metab 20(1)
: 91-110.

Mylchreest, E., et al. (2002) : Reprod Toxicol 16(1) : 19-28.

Fisher, J.S., et al. (2003) : Hum Reprod 18(7) : 1383-94.

Swan, S.H., et al. (2005) : Environ Health Perspect 113(8) : 1056-61.

Main, K.M., et al. (2006) : Environ Health Perspect 114(2) : 270-6.

Lambrot, R., et al. (2006) : JCEM $91: 2696-703$.

\section{POSTER 9}

Analyse de l'expression du gène de l'aromatase dans le spermatozoïde des hommes hypofertiles

\section{SAIID1,2, I. GALERAUD-DENIS2,3 , A. SAÂD1, L. BEAUGARD2, S. CARREAU2}

1 Service de Cytogénétique et Biologie de la Reproduction, Hôpital Universitaire Farhat Hached, Sousse, Tunisie ; 2 Département de Biochimie Université de Caen, France ;

3 Unité de Biologie de la Reproduction CHU Caen, France lamia_said2001@yahoo.fr

Introduction : Les estrogènes sont depuis longtemps considérés comme des hormones sexuelles typiquement femelles; cependant plusieurs travaux réalisés au cours de ces dernières années montrent qu'ils ont, aussi, un rôle important dans la fonction de reproduction mâle. L'aromatase est une enzyme qui transforme irréversiblement les androgènes en estrogènes. Une étude récente montre une diminution significative de l'expression du gène de cette enzyme dans les spermatozoïdes immobiles en comparaison aux spermatozoïdes mobiles. L'objectif de ce travail est d'estimer l'implication du gène de l'aromatase dans la qualité du spermatozoïde en ce qui concerne sa mobilité (asthénospermie) et sa morphologie (tératospermie) chez des tunisiens consultant pour infertilité.

Matériel et Méthodes : L'expression de gène de l'aromatase a été analysée sur 57 spermes provenant de 18 hommes normospermiques et 39 patients consultant pour infertilité au Service de Cytogénétique et Biologie de la Reproduction à l'hôpital Farhat Hached de Sousse. L'extraction des ARN totaux a été effectuée à partir de spermatozoïdes séparés sur gradient discontinu de Sil-Select. Après vérification de la qualité des ARN totaux extraits, une transcription inverse permettant la conversion des ARN totaux en ADNc a été réalisée pour tous les échantillons ensuite nous avons procédé à une PCR en temps réel pour l'analyse quantitative des taux de transcrits de l'aromatase. Pour chaque échantillon, un spermogramme et un spermocytogramme ont été aussi réalisés. Le logiciel StatView a été utilisé pour l'analyse statistique.

Résultats : Notre étude montre qu'il y a une diminution significative du taux des ARNm de l'aromatase aussi bien chez les tératospermiques $(52 \%)$ que chez les asthénotératospermiques (67\%) par rapport aux nomospermiques. Nos résultats indiquent aussi que les asthénospermiques constituent un groupe hétérogène en comparaison aux normospermiques. En effet, les asthénospermiques se divisent en deux sous-groupes avec des taux d'ARNm abaissés dans le premier et augmentés dans le second. De plus, notre étude met en évidence une corrélation significative entre les transcrits aromatase et le pourcentage de formes atypiques $(r=-0,64 ; p<$ 0,001 ) ou/et I'IAM (index d'anomalies multiples ; $r=-0,40, p<$ $0,01)$. Le coefficient de corrélation est plus élevé lorsque les anomalies morphologiques touchent la tête des spermatozoïdes plutôt que les flagelles. Ce sont les spermatozoïdes à acrosomes mal formés ou microcéphales qui semblent les plus concernés.

Conclusion : Le transcrit de l'aromatase semble constituer un bon marqueur de la qualité du spermatozoïde humain en relation avec 
la morphologie surtout les malformations de la tête ou la mobilité puisque la détection du transcrit est faible chez certains asthénospermiques et quasi impossible chez les asthénotératospermiques.

\title{
POSTER 10
}

Corrélations entre les taux des hormones sexuelles et la fonction sexuelle chez les patients avec hypogonadisme hypergonadotrophique

\author{
I. SLIM, T. MSAKNI, K. ACH, M. CAHDLI-CHAIEB, \\ S. TRIMECH-AJMI, A. MAAROUFI-BEIZIG, L. CHAIEB
}

Service d'Endocrinologie du Professeur L. Chaieb, CHU Farhat Hached, Sousse (Tunisie) ines.slim@yahoo.fr

Introduction : La sexualité a toujours occupé une part importante dans la qualité de vie des hommes. Peu d'études se sont intéressées à l'implication de la concentration des hormones sexuelles dans la qualité de vie sexuelle des patients en hypogonadisme. Le but de notre travail est d'évaluer la relation entre les caractéristiques cliniques et biologiques de l'hypogonadisme et l'activité sexuelle des patients avec hypogonadisme périphérique.

Patients et Méthodes : II s'agit d'une étude rétrospective incluant 62 patients suivis au service d'endocrinologie du CHU Farhat Hached entre janvier 1984 et Décembre 2005 pour hypogonadisme hypergonadotrophique congénital ou acquis. Les dossiers ne contenant pas d'informations valables sur la fonction sexuelle ont été éliminés ainsi que les patients diabétiques. Les données concernant les caractéristiques cliniques et biologiques de l'hypoandrogénie ainsi que la qualité de vie sexuelle ont été colligés.

Résultats : Parmi les 62 patients inclus, 40 avaient un hypogonadisme congénital (11 cas de Klinefelter et 25 cas d'hypogonadisme périphérique) et 22 patients avaient un hypogonadisme acquis (secondaire à un traumatisme, une chirurgie ou une radiotahérapie pelvienne dans 13 cas et 11 cas de déficit androgénique lié à l'âge). Soixante trois pourcent des pateints mariés ont eu spontanément des enfants (2,5 enfants en moyenne). Les patients ayant une stérilité primaire $(22 \%)$ ont consulté avec un retard diagnostique allant de 1 à 36 ans (Le cas extrême étant celui d'un patient ayant un syndrome de Klinefelter diagnostiqué à l'âge de 66 ans à l'occasion d'une consultation pour incidentalome hypophysaire). L'âge de découverte de l'hypogonadisme allaient de 19 à 81 ans (moyenne : $47,4 \pm 19$ ans). Les troubles sexuels ont constitué le motif de consultation dans $25,8 \%$ des cas alors que 17,7 $\%$ des patients ont consulté pour un problème de stérilité. Des signes cliniques d'hypoandrogénie ont été retrouvés dans $29 \%$ des cas. Une altération de la vie sexuelle a été objectivée dans $29 \%$ des cas et duraient en moyenne $\mathbf{4 3}$ mois avant la consultation. II s'agissait d'une impuissance complète dans $14,5 \%$ des cas, une anéjaculation dans $3,2 \%$ et une diminution de la libido dans $11,9 \%$ des cas. La testostérnémie moyenne était de $2,3 \pm 1,49 \mathrm{ng} / \mathrm{ml}$ et les taux ne corrélaient pas ni avec le degré d'hypoandrogénie clinique ni avec le degré d'altération de la vie sexuelle. Alors que l'âge de diagnostic était corrélé positivement et de façon significative $(p: 0,025)$ avec le degré d'altération de la fonction sexuelle.

Discussion et conclusion : Ce travail souligne l'importance de diagnostiquer précocement les hypogonadismes notamment congénitaux afin de les prendre en charge précocement et d'éviter une altération de la fonction sexuelle évolutive dans le temps. 